\title{
ОЦЕНКА МЕЖОТРАСЛЕВЫХ МУЛЬТИПЛИКАТИВНЫХ ЭФФЕКТОВ, ВЫЗЫВАЕМЫХ ЭКСПОРТНЫМИ ШОКАМИ
}

\section{В.А. Пархименко*}

Предложена методика оценки мультипликативных эффектов, вызываемых экспортными шоками, на основе методологии межотраслевого баланса Леонтьева и концепции мультипликатора внешней торговли Харрода. Методика позволяет учитывать специфику расходов домашних хозяйств, государства и коммерческих организаций, производить оценки в основных ценах и в ценах покупателей. Возможна оценка мультипликативных эффектов итерационно. Апробация методики осуществлена в рамках оценки отраслевых мультипликаторов валового выпуска, валовой добавленной стоимости и ВВП для китайской, российской и белорусской экономик.

Ключевые слова: межотраслевой баланс, методология «Затраты - Выпуск», мультипликатор Харрода, мультипликативные эффекты в экономике, экспортные шоки.

JEL-классификация: C50, C67, E12, E17.

DOI: $10.46782 / 1818-4510-2021-3-40-57$

Материал поступил 30.07.2021 2.

\section{Конщепщия мультипликатора во внешней торговле}

Для открытых экономик одними из важнейших факторов, определяющих их текущее состояние и рост в будущем, являются факторы внешнего порядка, относящиеся ко «всему остальному миру». В частности, в макроэкономических моделях часто в качестве таких внешних факторов используются уровень цен заграницы и заграничная ставка процента, а факторов, тесно связанных с конъюнктурой мировых рынков, - обменный курс национальной валюты, сальдо платежного баланса.

Изменение в объеме экспортируемых товаров и услуг отечественного происхождения при прочих равных - один из таких факторов, который является объектом настоящего исследования. Колебания в объемах товаров и услуг, поставляемых за рубеж, осуществляются постоянно, однако в данной статье мы особое внимание уделяем резким (неожидаемым) и существенным изменениям, сохраняющимся длительное время, в первую очередь - в сторону умень- шения, так называемым «шокам». Примерами таких экспортных шоков могут быть изменение цен на мировых рынках (яркий пример - нефть), введение режима «локдауна» ввиду пандемии COVID-19 в странах клиентах экспортируемой продукции, экономические санкции, военные конфликты в мире и т. п.

Первоначальными (прямыми) эффектами экспортных шоков очевидным образом выступают сокращение объемов производства непосредственно экспортируемых товаров и услуг, валовой добавленной стоимости в отраслях-экспортерах и, как результат, падение ВВП.

Существуют и косвенные эффекты. В первую очередь это сокращение производства товаров и услуг, которые входят в экспортируемую продукцию в качестве комплектующих или другим образом используются при их производстве. Например, устойчивое и существенное падение в объемах экспорта автотракторной техники вызовет сокращение выпуска двигателей внутреннего сгорания и шин, что, в свою оче-

* Пархименко Владимир Анатольевич (parkhimenko@bsuir.by), кандидат экономических наук, доцент, Белорусский государственный университет информатики и радиоэлектроники (г. Минск, Беларусь). https://orcid.org/0000-0001-7690-8873 
редь, повлияет на выпуск в таких отраслях, как металлургическая и производство резиновых изделий.

Вследствие косвенного влияния экспортного фактора по всем затронутым национальным цепочкам создания стоимости произойдет уменьшение доходов, валовой добавленной стоимости, занятости, уровня загрузки производственных мощностей.

Однако существуют и вторичные (индуцированные) эффекты, которые мы называем мультипликативными. Речь идет о том, что уменьшение объемов выпуска в затронутых прямо или косвенно отраслях приводит к уменьшению доходов в этих отраслях - заработной платы занятых работников (домохозяйств), прибыли коммерческих организаций, налогов, идущих в бюджет государства, что, в свою очередь, при прочих равных приводит к последующему сокращению расходов этих субъектов на конечные товары и услуги, т. е. к падению совокупного спроса, теперь уже не внешнего, как в случае экспорта, а внутреннего. Новое же падение конечного спроса внутри страны вызывает новую «волну» сокращений выпуска по соответствующим отраслям, валовой добавленной стоимости, доходов и очередное падение внутреннего спроса.

Подобные прямые, косвенные и мультипликативные эффекты обычно могут быть оценены в агрегированном виде на макроэкономическом уровне посредством кейнсианской модели «мультипликатора», учитывающей первоначальный и все последующие приросты национального дохода, вызванные приростом тех или иных компонентов валового внутреннего продукта. При этом такие компоненты считаются независящими напрямую от национального дохода и поэтому маркируются термином «автономные».

Мультипликатор автономных расходов в кейнсианской традиции, как известно, рассчитывается исходя из предельной склонности к потреблению домашних хозяйств, ставки подоходного налога и предельной склонности к потреблению импортных благ ${ }^{1}$ :

${ }^{1}$ Тарасевич Л.С., Гальперин В.М., Гребенников П.И., Леусский А.И. 1999. Макроэкономика: учебник. СПб.: Издво СПбГУЭФ. С. 78.

$$
\Delta y=\frac{1}{1-C_{y}+C_{y} T_{y}+Z_{y}} \Delta A,
$$

где $\Delta y-$ изменение реальной величины национального дохода;

$C_{y}$ - предельная склонность к потреблению располагаемого после уплаты налогов дохода;

$T_{y}$ - ставка подоходного налога;

$Z_{y}$ - предельная склонность к потреблению импортных благ;

$\Delta A$ - изменение автономных расходов ${ }^{2}$.

В формуле (1) непосредственно мультипликатором является дробь, стоящая слева перед $\triangle A$.

Говоря о концепции кейнсианского мультипликатора, которую, как считается, впервые предложил даже не Дж.М. Кейнс, а Ричард Кан ${ }^{3}$, нельзя не коснуться существующей критики этого подхода ${ }^{4}$ В частности, в литературе упоминаются следующие положения, ставящие под сомнение практическую значимость концепции мультипликатора: мультипликатор не учитывает временной аспект (лаги) макроэкономических процессов; мультипликатор не учитывает динамический (меняющийся) характер макроэкономических процессов; мультипликатор не имеет полноценного эмпирического подтверждения; мультипликатор делает упор на конечное потребление домашних хозяйств, нивелируя значимость инвестиций и государственных расходов; мультипликатор не учитывает зависимость инвестиций от дохода; мультипликатор предполагает слишком жесткие, однозначные, «механические» связи в экономике ${ }^{5}$.

2 Изменение автономных расходов может быть как положительным, так и отрицательным - мультипликатор «работает» в обе стороны. Однако в учебниках по макроэкономике, как правило, приводятся примеры роста расходов и, как следствие, роста национального дохода, что соответствует общему посылу Дж. М. Кейнса о необходимости активного вмешательства государства в случаях низкого совокупного спроса. В контексте данной статьи, напротив, больший интерес представляет мультипликативное падение национального дохода в случае экспортных шоков.

${ }^{3}$ Kahn R. 1931. The Relation of Home Investment to Unemployment. The Economic Journal. Vol. 41. No 162. PP. 173-198.

${ }^{4}$ См., например, Ahiakpor (2001).

${ }^{5}$ URL: https://www.yourarticlelibrary.com/theories/6drawbacks-of-multiplier-theory-formulated-by-keynes/25608 
Кроме того, следует помнить, что мультипликатор предполагает переход экономики из одного равновесного состояния в другое при неизменном уровне цен, т. е. лишь на основе количественного приспособления без участия ценового механизма ${ }^{6}$. Наконец, концепция мультипликатора берет в рассмотрение только совокупный спрос, полагая предложение бесконечно расширяемым. Оба эти предположения нельзя признать реалистичными.

И все же, несмотря на озвученные слабые стороны концепции мультипликатора, практически все современные учебники по экономической теории и макроэкономике содержат материал, посвященный мультипликативным эффектам и мультипликаторам кейнсианского вида, например как в учебниках представителей так называемого мейнстрима (Mankiw, 2019), так и в учебниках представителей неортодоксальной современной денежной теории (Mitchell, Wray, Watts, 2019).

В прикладном анализе, однако, в настоящее время для оценки мультипликативных эффектов применяется не классическая формула, более подходящая для объяснения самых простых макроэкономических концепций в образовательном процессе, а эконометрические методы, например векторная модель коррекции ошибок с экзогенными переменными (VECMX). Подобный подход позволяет на основе временных рядов отыскать статистически значимые взаимосвязи (как правило, с учетом временных лагов) между приростом расходов, с одной стороны, и валовым внутренним продуктом, с другой.

В любом случае можно констатировать, что понятие мультипликативных эффектов входит в лексикон современных экономистов, пусть даже конкретное содержательное наполнение этого термина может варьироваться.

\section{Мультипликатор Харрода}

Как известно, в кейнсианстве основной фокус внимания - краткосрочная мак-

${ }^{6}$ Тарасевич Л.С., Гальперин В.М., Гребенников П.И., Леусский А.И. 1999. Макроэкономика: учебник. СПб.: Издво СПбГУЭФ. С. 81. роэкономическая динамика. Именно поэтому крайне важным является учет того факта, что не все сбережения в краткосрочном периоде становятся инвестициями, а получаемые государством налоговые доходы - государственными расходами. И в таком случае речь идет об «утечке» (leakage) этих средств, т. е. их «выпадении» в краткосрочном периоде из экономического процесса.

В предлагаемой ниже методике в отличие от традиционного кейнсианского подхода в качестве автономного компонента конечного (совокупного) спроса рассматриваются лишь экспортные поставки отечественных товаров и услуг, а государственные и инвестиционные расходы выступают как функционально зависящие от национального дохода.

Подобный подход, несмотря на некоторое упрощение реальных макроэкономических процессов, ориентирован на то, чтобы оценить, каково влияние экспортных шоков на прирост валового внутреннего продукта при условии, что конечное потребление, валовое накопление основного капитала и изменение запасов материальных оборотных средств напрямую (линейно) связано с приростом или падением доходов от экспорта.

Таким образом, предлагаемый подход оценивает потенциальное влияние экспортных шоков при условии сохранения сложившихся в экономике межотраслевых пропорций, неизменности стратегии поведения домашних хозяйств, государства и инвесторов.

Пожалуй, будет правильным сказать, что озвученный подход развивает идеи, заложенные еще в 1933 г. ${ }^{7}$ в мультипликаторе международной торговли Харрода, в соответствии с которым рост объемов экспорта вызывает рост национального дохода с мультипликатором, определяемым через предельную склонность к импорту (Thirlwall, 2012):

$$
\Delta y=\frac{1}{Z_{y}} \Delta E
$$

где $\Delta E$ - изменение величины экспорта.

${ }^{7}$ Harrod R. 1933. International Economics. Cambridge: Cambridge University Press. 
Мультипликатор Харрода по сути фиксирует очень простую логику ${ }^{8}$. Допустим, экспорт изначально вырос на некоторую величину $\Delta E$, т. е. в страну «притекли» дополнительные доходы из-за границы. Часть этих доходов (в размере $Z_{y}$ на каждый рубль дополнительных доходов) будет в дальнейшем потрачена на закупку импортных товаров и услуг, а оставшаяся часть $\left(1-Z_{y}\right)$ пойдет на закупку отечественных товаров и услуг. На следующем шаге вновь одна часть $\left(Z_{y}\right)$ «утечет» за границу, а на внутреннем рынке будет потрачена часть, равная $\left(1-Z_{y}\right)\left(1-Z_{y}\right)=\left(1-Z_{y}\right)^{2}$. Процесс в теории будет повторяться бесконечно. В развернутом виде математически формулу (2) можно записать следующим образом с учетом свойств бесконечно убывающей геометрической прогрессии:

$$
\begin{aligned}
& \Delta y=\Delta E+\Delta E\left(1-Z_{y}\right)+\Delta E\left(1-Z_{y}\right)^{2}+\ldots+\Delta E\left(1-Z_{y}\right)^{n}= \\
& =\left(1+\left(1-Z_{y}\right)+\left(1-Z_{y}\right)^{2}+\ldots+\left(1-Z_{y}\right)^{n}\right) \Delta E= \\
& =\frac{1}{Z_{y}} \Delta E \text { для } n \rightarrow \infty .
\end{aligned}
$$

Конечно, мультипликатор Харрода является существенным упрощением реальной действительности.

Во-первых, он предполагает, как указывалось выше, отсутствие автономности инвестиционных и государственных расходов, т. е. наличие прямой зависимости между национальным доходом и указанными расходами. Так, к примеру, все сбережения конвертируются в инвестиции, а все нало-

8 Сам Харрод в $\S 1$ 6-й главы своей монографии явным образом не говорит о мультипликаторе или мультипликативных эффектах как таковых, а использует выражение «равновесие в упрощенных условиях» (equilibrium in simplified conditions). Тем не менее он исходит из той базовой посылки, что для того, чтобы были возможны расходы на импортируемые товары и услуги, необходимы равновеликие доходы от экспортной деятельности. В случае, когда экспортные доходы падают, падают и расходы на отечественные товары (товары С в терминологии Харрода) и импортные товары (товары А и В). Падение расходов на отечественные товары означает падение доходов отечественных отраслей и новое уменьшение расходов на отечественные и импортные товары. Этот процесс продолжается до тех пор, пока падение объема импорта не сравнивается с первоначальным падением экспорта, т. е. экономика переходит в новое состояние равновесия, при котором экспорт равен импорту (Harrod, 1933. PP. 110-111). говые доходы - в государственные расходы (McCombie, 1985).

Во-вторых, он базируется на допущении, что сальдо текущего счета платежного баланса страны всегда равно нулю, т. е. экспорт всегда равен импорту.

В-третьих, предельная склонность к импорту $\left(Z_{y}\right)$ оценивается (Thirlwall, 2012) на базе линейной регрессии вида $Z=Z_{a}+Z_{y} \cdot y$, где $Z_{a}$ - объем автономного импорта, не зависящего от национального дохода. Линейность такой зависимости, ее устойчивость во времени, отсутствие дифференциации между конечным импортом и промежуточным, а также учета разницы между импортоемкостью экспорта и внутреннего спроса и лага между доходами и импортом - очевидные спорные места данного подхода.

В-четвертых, представляется, что мультипликатор несколько завышает оценку вклада прироста экспорта в национальный доход, так как согласно (3) первоначальный прирост $\Delta E$ целиком входит в состав прироста национального дохода, хотя на самом деле лишь добавленная стоимость национального происхождения, содержащаяся в стоимости экспортируемых товаров и услуг, войдет в это значение.

B-пятых, при полностью закрытой экономике (импорт равен нулю) мультипликатор Харрода становится равным +ю. А так как у Харрода предполагается равенство экспорта импорту, то это может быть сформулировано и таким образом, что при все большем уменьшении доли экспорта в ВВП мультипликатор внешней торговли бесконечно возрастает. Подобное сложно поддается какой-либо осмысленной интерпретации.

Тем не менее, несмотря на несколько упрощенное моделирование реальных экономических процессов, мультипликатор Харрода представляется весьма ценным способом фиксации структурных особенностей экономики, характеризующих ее принципиальную «отзывчивость» на экспортные шоки.

Развитие положений Харрода, осуществленное Тирлволлом в 1979 г. (Thirlwall, 1979) и обычно именуемое в литературе как динамический мультипликатор Харрода или закон Тирлволла, базируется на более реалистичных предпосылках (в частности, ис- 
пользуется нелинейная функция импорта) и неоднократно подвергалось успешной эмпирической проверке (Bairam, Dempster, 1991). Закон Тирволла связывает темп роста национального дохода (ВВП) с темпом роста экспорта и эластичностью импорта по доходу и позволяет получить количественную оценку такого максимально возможного роста, который не приводит к дисбалансам платежного сальдо.

Если оценить значения классического (статического) мультипликатора Харрода для некоторых стран мира, используя данные Всемирного банка по национальному доходу и импорту за 1960-2020 гг., получится следующая картина (табл. 1).

Отметим при этом, что:

- национальный доход в табл. 1 для всех стран брался в долларах США в текущих ценах, рассчитанный по методу Атла$\mathrm{ca}^{9}$, данные по импорту также в текущих ценах в долларах США;

- для большинства стран данные по импорту были доступны не за весь рассматриваемый период, а лишь за определенные годы, что отмечено во второй графе табл. 1;

${ }^{9}$ URL: https://datahelpdesk.worldbank.org/knowledge base/articles/378832-the-world-bank-atlas-method-detailedmethodology
- оценка линейной регрессии производилась в Rstudio посредством функции $\operatorname{lm}()$;

- несмотря на очевидное наличие структурных сдвигов во всех рассматриваемых экономиках на таком большом промежутке времени, коэффициенты линейной регрессии оказались статистически значимыми на уровне $p<0,001$, а коэффициент детерминации высоким $\left(R^{2}>0,92\right.$ для 11 стран из выборки и $R^{2}>0,79$ для двух оставшихся), что в общем и целом свидетельствует о довольно устойчивой и непосредственной связи между национальным доходом и импортом.

Интерпретация полученных оценок должна осуществляться с большой осторожностью и учетом упомянутых выше слабостей концепции мультипликатора в целом и особенностей подхода Харрода в частности.

Тем не менее вполне очевидно, что в некоторых рассмотренных странах (Япония, Бразилия, США) косвенный (мультипликативный) эффект от изменения экспортной выручки отечественных компаний потенциально существенно выше, чем в других (Беларусь, Германия, Южная Корея), и это напрямую связано со структурой экономики, ее меньшей зависимостью от импортных товаров и услуг и большей ориентацией на внутреннее потребление.

Таблица 1

Оценка значений статического мультипликатора Харрода для некоторых стран мира, долл. США (в текущих ценах)

\begin{tabular}{|l|c|c|c|c|}
\hline \multicolumn{1}{|c|}{ Страна } & Период & $\begin{array}{c}\text { Предельная } \\
\text { склонность } \\
\text { к импорту }\end{array}$ & $R^{2}$ & $\begin{array}{c}\text { Мультипликатор } \\
\text { Харрода }\end{array}$ \\
\hline Япония & $1970-2019$ & $0,136^{* * * *}$ & 0,787 & 7,35 \\
\hline Бразилия & $1982-2020$ & $0,146^{* * *}$ & 0,977 & 6,85 \\
\hline США & $1970-2019$ & $0,167^{* * *}$ & 0,983 & 5,99 \\
\hline Китай & $1962-2020$ & $0,190^{* * *}$ & 0,960 & 5,26 \\
\hline Россия & $1989-2020$ & $0,201^{* * *}$ & 0,916 & 4,98 \\
\hline Индия & $1962-2020$ & $0,251^{* * *}$ & 0,950 & 3,98 \\
\hline Италия & $1970-2020$ & $0,258^{* * *}$ & 0,947 & 3,88 \\
\hline Канада & $1962-2020$ & $0,288^{* * *}$ & 0,960 & 3,47 \\
\hline Франция & $1962-2020$ & $0,292^{* * *}$ & 0,972 & 3,42 \\
\hline Великобритания & $1970-2020$ & $0,309^{* * *}$ & 0,980 & 3,24 \\
\hline Корея & $1962-2020$ & $0,395^{* * *}$ & 0,947 & 2,53 \\
\hline Германия & $1970-2020$ & $0,401^{* * *}$ & 0,979 & 2,49 \\
\hline Беларусь & $1994-2020$ & $0,658^{* * *}$ & 0,841 & 1,52 \\
\hline
\end{tabular}

*** Уровень статистической значимости $p<0,001$.

Источник. Рассчитано по данным Всемирного банка: URL: https://api.worldbank.org/v2/en/indicator/ NY.GNP.ATLS.CD?downloadformat=excel; URL: https://api.worldbank.org/v2/en/indicator/NE.IMP.GNFS.CD? downloadformat=excel 
Подобные результаты выглядят несколько парадоксально. Например, в такой открытой экономике, как у Республики Беларусь, несмотря на очевидную зависимость от внешнеторговой деятельности, значение мультипликатора Харрода невысокое. А, например, в относительно закрытой экономике Японии мультипликатор Харрода практически в 5 раз больше. На первый взгляд получается, что на закрытые экономики экспорт воздействует намного больше.

Это объясняется тем, что в открытой экономике экспорт в основном оказывает влияние на ВВП через прямые, а не косвенные (мультипликативные) эффекты. При этом эффекты ощущаются непосредственно, противодействовать им довольно сложно, так как нужно оперативно компенсировать «выпадающий» экспорт экспортом в какие-то новые страны. В более закрытых экономиках прямые эффекты не столь значительны, однако они потенциально могут посредством мультипликативных процессов еще долгое время оказывать влияние на экономику, правда, им можно противодействовать до определенной степени мерами стимулирования внутреннего спроса.

\section{Межотраслевой подход}

Мультипликатор Кейнса, как и мультипликатор Харрода, это мультипликаторы скалярного вида, они представляют макроэкономические процессы распространения первоначального импульса (экспорта или иных автономных расходов) в агрегированном виде, по всей экономике в целом, без детализации этих процессов и их дифференциации по различным секторам и отраслям.

В научной литературе встречаются упоминания об использовании межотраслевого подхода (модель «Затраты - Выпуск» В.В. Леонтьева) для получения более детализированной картины при оценке мультипликативных эффектов, порождаемых в сфере внешней торговли или изменениями иных компонентов ВВП.

С помощью классической модели Леонтьева, позволяющей рассчитать все полные затраты на производство единицы той или иной конечной продукции, могут быть достаточно неплохо оценены косвенные эффекты экспортных шоков.

Однако так называемая открытая модификация модели Леонтьева, в которой конечный спрос является величиной экзогенной, не позволяет зафиксировать вторичные эффекты (в целях данной статьи мы называем их мультипликативными). Речь идет о том, что уменьшение объемов выпуска в затронутых прямо или косвенно отраслях приводит к уменьшению доходов в этих отраслях - заработной платы занятых работников (домохозяйств), прибыли коммерческих организаций, налогов, идущих в бюджет государства, что, в свою очередь, при прочих равных вызывает последующее сокращение расходов этих субъектов на конечные товары и услуги, т. е. падение совокупного спроса, теперь уже не внешнего, как в случае экспорта, а внутреннего. В свою очередь, такое новое падение конечного спроса внутри страны приводит к новой «волне» сокращений выпуска по соответствующим отраслям, валовой добавленной стоимости, доходов и новому падению внутреннего спроса.

В вариациях модели Леонтьева, в которых эндогенизируются различные компоненты конечного спроса (в первую очередь расходы домашних хозяйств), подобные эффекты учитываются в итоговом решении. Это, например, модель Миядзавы (Miyazawa, 1960; 1968; 1976) и ее производные версии.

Стоит отметить, что 6 глава классического учебника Миллера и Блэра (Miller, Blair, 2009) по методологии «Затраты Выпуск» (как в его текущем 2009 г. издании, так и в его планируемом 3-м издании в октябре 2021 г.) целиком посвящена мультипликаторам. Отметим при этом, что термин «мультипликаторы» в данном случае неоднозначен, так как в методологии «Затраты - Выпуск» мультипликаторами называют разные коэффициенты, которые показывают прирост тех или иных показателей в ответ на изменение конечного спроса. В первую очередь это мультипликаторы валового выпуска, учитывающие полные затраты из обратной матрицы Леонтьева. Есть также мультипликаторы, показывающие прирост потребности в импорте на дополнительную единицу конечного спро- 
са (в отечественной литературе в этом случае обычно говорят не о мультипликатоpax, a о полной импортоемкости). Однако в методологии «Затраты - Выпуск» речь идет также и о мультипликаторах, учитывающих индуцированные эффекты, т. е. те, которые возникают из-за прироста доходов домашних хозяйств и последующего увеличения расходов на потребление именно эти мультипликаторы интересны в контексте данной статьи. Как правило, в литературе используется следующая классификация: Туре I - «обычные» мультипликаторы Леонтьева, учитывающие прямые и косвенные эффекты при изменении конечного спроса, и Туре II - мультипликаторы, учитывающие еще и вторичные (индуцированные, мультипликативные) эффекты, вызываемые приростом доходов домохозяйств и последующим приростом конечных расходов.

Если обратиться к истории, то сама идея мультипликатора не в скалярном виде (числе), а в матричном была высказана довольно давно. Godwin еще в 1949 г. предложил трансформировать мультипликатор Кейнса, учитывающий агрегированные по экономике показатели, в мультипликатор матричного вида на основе подхода «затраты-выпуск» Леонтьева, тем самым получая возможность определить независимые мультипликаторы по каждой из отраслей, а также ввести в рассмотрение динамический аспект распространения мультипликативного эффекта по экономике $\mathrm{e}^{10}$.

Миядзава говорит о недостатках подхода Кейнса к анализу мультипликативного эффекта внешней торговли (не совсем корректный учет конечного и промежуточного импорта) и той разновидности модели Леонтьева, в которой домашние хозяйства представлены в виде фиктивной производящей отрасли. Миядзава предлагает ввести функцию потребления домашних хозяйств кейнсианского типа в модель «Затраты - Выпуск» и выводит формулу матричного мультипликатора внешней торговли (Miyazawa, 1960).

В диссертационной работе Bonnici напрямую не использует концепцию мульти-

${ }^{10}$ Goodwin R. 1949. The Multiplier as Matrix. The Economic Journal. Vol. 59. No 236. PP. 537-555. пликатора, однако при построении макроэкономической модели для Мальты на основе модели «Затраты-Выпуск» опирается на кейнсианскую функцию потребления (в частности, рассматривает подход Миядзавы в этом контексте) и показывает, что такая модель на исторических данных дает более точные прогнозы, чем стандартный подход Леонтьева ${ }^{11}$. Спустя 32 года Gravino (2012) произвел для экономики Мальты оценки мультипликаторов двух упоминаемых выше типов - Type I и Type II. Gravino показывает, что мультипликаторы второго типа для всех отраслей экономики Мальты ожидаемо больше, чем мультипликаторы первого типа. Несмотря на невозможность провести прямую оценку результатов Bonnici и Gravino, о чем пишет последний, их подходы являются примерами прикладного использования комбинации межотраслевой методологии и кейнсианской функции потребления.

Следует отметить, что модель «Затраты - Выпуск» Леонтьева - не единственный инструментарий исследователей для оценки мультипликатора внешней торговли. Так, используют подход Пазинетти (multi-sectoral macrodynamic analysis), он также базируется на таблицах «Затраты Выпуск» (Araujo, Lima, 2007), однако учитывает разные эластичности по доходу для разных продуктов, к тому же изменяющиеся в зависимости от роста дохода на душу населения, что приводит к нелинейной динамике конечного спроса и возникающих вследствие этого эффектов. На основе модели Пазинетти авторы формулируют так называемый межотраслевой закон Тирлволла (Multi-Sectoral Thirlwall's Law), который они, как и сам Тирлволл, рассматривают как динамическую версию мультипликатора Харрода. Правда, они так и остаются в сфере теории, не предлагая эмпирической апробации их идей.

Применение межотраслевого подхода для оценки классического (статического) мультипликатора Харрода на основе открытой модели Пазинетти предлагается и в публикации (Trigg, Ricardo, 2014), в кото-

${ }^{11}$ Bonnici J. 1980. Integrating Input-Output and Keynesian Models: A Case Study of Malta. Unpublished doctoral dissertation. Canada: Simon Fraser University. 
рой авторы в качестве полезного прикладного результата своего подхода рассматривают необходимость реструктуризации экономики, в частности перемещения ресурсов страны из отраслей с высокой эластичностью по доходу спроса на импорт в отрасли с низкой эластичностью с целью увеличения ВВП. Как и в публикации выше, авторы представляют только теоретическую модель, без осуществления прикладных оценок по конкретным экономикам. Такие расчеты сделаны другими авторами (Gouvea, Lima, 2010; Gouvea, Lima, 2013; Romero, McCombie, 2016), однако их основной целью было подтверждение того, что расчетные значения равновесного роста по закону Тирлволла адекватны фактическим значениям.

Интерес представляет подход, предложенный российскими исследователями и апробированный на данных таблиц «Затраты - Выпуск» для РФ за 2014 г. (Ксенофонтов, Широв, Ползиков, Янтовский, 2018). Авторы, используя статическую модель Леонтьева, предлагают оценивать лишь первый цикл индуцированных (мультипликативных) эффектов, что существенно отличается от, например, подхода Миядзавы, где такое количество циклов бесконечно. Еще одним отличием является оценивание мультипликативного эффекта, вызываемого приростом не только расходов домашних хозяйств, но и иных субъектов - государства и коммерческих организаций.

Для российской экономики предложен и другой подход, заслуживающий внимание, в котором Дондоковым и Булдаевым разработана модель баланса суммарных расходов (МОБСР), являющаяся синтезом классической модели межотраслевого баланса и кейнсианской модели мультипликатора. В частности, авторы выделяют мультипликатор полных внутренних материальных затрат (мультипликатор Леонтьева), мультипликатор суммарных расходов отечественной продукции, отраслевой мультипликатор дохода (Дондоков, Булдаев, 2013).

Подводя итог краткому обзору литературы, следует отметить многолетний устойчивый интерес к межотраслевой оценке мультипликативных эффектов в экономике, вызываемых в том числе динамикой экспорта. При этом в качестве базовой методологии используется либо модель Леонтьева, либо модель Пазинетти. Насколько можно судить, наиболее популярным у исследователей является стандартный кейнсианский подход к определению мультипликатора как с точки зрения доминирующей роли потребления домашних хозяйств, так и с точки зрения «утечек» (leakage) в использовании национального дохода в виде сбережений, налогов и импорта. Такие авторы рассматривают инвестиционные и государственные расходы как автономные (не зависящие от национального дохода), поэтому в качестве «замыкающего» фактора берут лишь потребление домашних хозяйств. Та часть национального дохода, которая «утекает» в виде сбережений, налогов и покупки импортных товаров и услуг, при подобном подходе не трансформируется автоматически в инвестиционные и государственные расходы в составе конечного спроса. Тем не менее встречаются оценки непосредственно мультипликатора Харрода в статической и динамической спецификации.

Важной видится задача предложить методику оценки мультипликативных эффектов в экономике, которая бы: 1) позволяла оценить максимально возможный мультипликативный эффект, т. е. использовала логику подхода Харрода, а не Кейнса; 2) учитывала методологию и существующие таблицы «Затраты - Выпуск» с их спецификой у конкретных стран; 3) позволяла при необходимости оценивать мультипликативные эффекты итерационно, т. е. по каждому шагу в отдельности; 4) позволяла производить оценки не только в основных ценах, но и с учетом чистых налогов на продукты и торговых и транспортных наценок; 5) позволяла учитывать специфику расходов домашних хозяйств, государства и коммерческих организаций.

\section{Методика оценки межотраслевых мультипликативных әффектов упрощенным образом на основе валовой добавленной стоимости}

Для перехода от мультипликатора в скалярной форме (числа) к более детализированному способу фиксации мультипликатив- 
ных эффектов посредством модели межотраслевого баланса можно предложить следующую логическую схему расчетов (рис. 1).

Рассмотрим методику расчетов, реализующих эту общую логическую схему. При этом будем базироваться на модели Леонтьева, учитывающей раздельным образом прямые затраты отечественных и импортных товаров и услуг и описанной в более ранних публикациях автора (Быков, Пархименко, Толкачев, 2020).

Пусть первоначальный импульс в виде экспортного шока вызывает изменение конечного спроса на отечественные товары и услуги и описывается вектором-столбцом $\Delta Y_{0}$, тогда необходимый прирост валового выпуска $\Delta X_{0}$ (вектор-столбец) будет равен

$$
\Delta X_{0}=B \cdot \Delta Y_{0}
$$

где $B$ - матрица полных затрат отечественных товаров и услуг, или матрица Леонтьева, получаемая из матрицы прямых затрат отечественных товаров и услуг: $B=\left(E-A_{\text {отеч }}\right)^{-1}$.

Отсюда следует, что прирост валовой добавленной стоимости $\left(\Delta V A_{0}\right)$ составит

$$
\Delta V A_{0}=V \cdot \Delta X_{0}=V \cdot B \cdot \Delta Y_{0}
$$

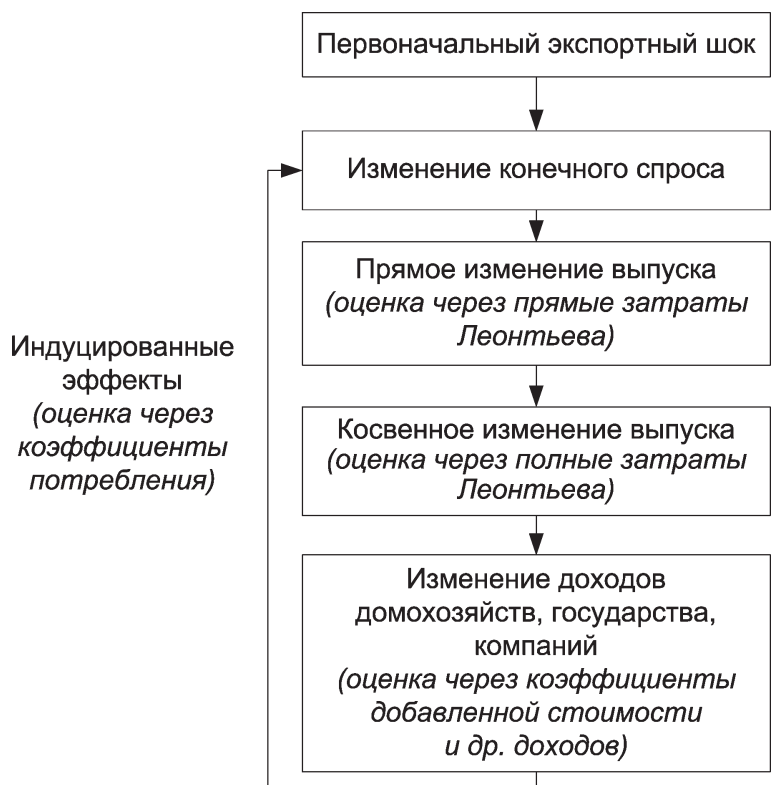

Рис. 1. Общая логическая схема методики расчетов

Источник. Авторская разработка. где $V$ - матрица (в простейшем случае вектор-строка), описывающая долю валовой добавленной стоимости в валовом выпуске.

Прирост валовой добавленной стоимости представляет собой доходы разных субъектов экономики, получаемые в форме заработной платы, чистой прибыли и смешанного дохода, налогов. В самом простом случае рассмотрим эти доходы без дифференциации, т. е. просто как суммарный доход, который далее расходуется на конечное потребление внутри страны - на покупку отечественных и импортных товаров и услуг. Таким образом полученные на первом шаге дополнительные доходы приведут к новому приросту конечного потребления.

Пусть $C$ - матрица (в простейшем случае - вектор-столбец), которая содержит коэффициенты потребления, показывающие потребление отечественных товаров и услуг, приходящееся на 1 руб. валовой добавленной стоимости, получаемой субъектами национальной экономики в виде доходов. Коэффициент по $i$-й отрасли будет, соответственно, равен в общем виде

$$
c_{i}=\frac{h_{i}^{\text {отеч }}+g_{i}^{\text {отеч }}+i n v_{i}^{\text {отеч }}}{V A},
$$

где $h_{i}^{\text {отеч }}+g_{i}^{\text {отеч }}+i n v_{i}^{\text {отеч }}-$ соответственно расходы домашних хозяйств, государственных учреждений, коммерческих организаций на конечное потребление отечественных товаров и услуг;

$V A$ - сумма валовой добавленной стоимости.

C учетом того, что по фактическим данным межотраслевого баланса сумма валовой добавленной стоимости и фактические расходы на потребление, как правило, отличаются ввиду, например, использования сбережений прошлых лет, расчет $c_{i}$ можно осуществлять несколько иным образом использовать в знаменателе общую сумму расходов на потребление:

$$
c_{i}=\frac{h_{i}^{\text {отеч }}+g_{i}^{\text {отеч }}+i n v_{i}^{\text {отеч }}}{\sum_{1}^{m}\left(h_{i}+g_{i}+i_{i}\right)},
$$

где $m-$ количество отраслей; 
$h_{i}+g_{i}+i_{i}-$ сумма расходов на конечное потребление на внутреннем рынке по $i$-й отрасли.

Остается открытым вопрос, в каких ценах следует брать значения в формулах (6) и (6') - в основных или в ценах покупателя. Представляется правильным для оценки коэффициентов по формуле (6) брать значения в основных ценах, а для оценки по формуле (6') - в ценах покупателей.

Зная значения элементов матрицы (вектора-столбца) $C$, можно оценить новый (индуцированный) прирост конечного спроса $-\Delta Y_{1}$ :

$$
\begin{aligned}
\Delta Y_{1} & =C \cdot \Delta V A_{0}=C \cdot V \cdot \Delta X_{0}= \\
& =C \cdot V \cdot B \cdot \Delta Y_{0} .
\end{aligned}
$$

Далее описанные выше итерации повторяются. Так, $\Delta Y_{2}$ будет равен

$$
\begin{aligned}
& \Delta Y_{2}=C \cdot \Delta V A_{1}=C \cdot V \cdot \Delta X_{1}=C \cdot V \cdot B \cdot \Delta Y_{1}= \\
& =C \cdot V \cdot B \cdot C \cdot V \cdot B \cdot \Delta Y_{0}=(C \cdot V \cdot B)^{2} \cdot Y_{0} .
\end{aligned}
$$

Для $n$-го шага, таким образом, получим:

$$
\Delta Y_{n}=(C \cdot V \cdot B)^{n} \cdot \Delta Y_{0} .
$$

А прирост валовой добавленной стоимости для $n$-го шага будет

$$
\Delta V A_{n}=V \cdot B \cdot(C \cdot V \cdot B)^{n} \cdot \Delta Y_{0} .
$$

Отметим, что формулы (9) и (10) позволяют получить значения приростов для любого шага и тем самым осуществлять расчет итерационно.

Для оценки суммарного мультипликативного эффекта нас интересует сумма всех приростов конечного спроса:

$$
\sum_{n=0}^{\infty} \Delta Y_{n}=\sum_{n=0}^{\infty}(C \cdot V \cdot B)^{n} \cdot \Delta Y_{0}
$$

Так как матричный ряд

$$
\begin{aligned}
\sum_{n=0}^{\infty}(C \cdot V \cdot B)^{n} & =E+C \cdot V \cdot B+(C \cdot V \cdot B)^{2}+ \\
& +(C \cdot V \cdot B)^{3}+\ldots
\end{aligned}
$$

при его сходимости и соблюдении условия Хокинса - Саймона дает матрицу вида $(E-C \cdot V \cdot B)^{-1}$, можно записать

$$
\sum_{n=0}^{\infty} \Delta Y_{n}=(E-C \cdot V \cdot B)^{-1} \cdot \Delta Y_{0} .
$$

Матрица $M_{Y}$ при этом будет описывать матричный мультипликатор конечного спроса:

$$
M_{Y}=(E-C \cdot V \cdot B)^{-1} .
$$

Каждый элемент матрицы $M_{Y}-$ возьмем, к примеру, $i j$-й элемент - показывает, на сколько денежных единиц вырастет конечный спрос на $i$-й продукт при росте конечного спроса на $j$-й продукт на 1 денежную единицу.

Из (10) и (12) следует, что для валовой добавленной стоимости будет справедливо следующее выражение:

$$
\begin{aligned}
& \sum_{n=0}^{\infty} \Delta V A_{n}=\sum_{n=0}^{\infty} V \cdot B \cdot \Delta Y_{n}=V \cdot B \cdot \sum_{n=0}^{\infty} \Delta Y_{n}= \\
& =V \cdot B \cdot(E-C \cdot V \cdot B)^{-1} \cdot \Delta Y_{0}=M_{V A} \cdot \Delta Y_{0},
\end{aligned}
$$

где $M_{V A}$ - матрица (в простейшем случае вектор-строка), содержащая значения мультипликаторов валовой добавленной стоимости по каждой из отраслей, соответственно, равная

$$
M_{V A}=V \cdot B \cdot M_{Y} .
$$

Элемент $j$ вектора-строки (для простейшего случая) $M_{V A}$ показывает, как вырастет валовая добавленная стоимость по экономике в целом при росте конечного спроса на отечественные товары и услуги $i$-й отрасли на единицу.

Наконец, можно также рассчитать матрицу $M_{X}$ - матричный мультипликатор валового выпуска:

$$
M_{X}=B \cdot M_{Y},
$$

где $i j$-й элемент показывает, на сколько денежных единиц вырастет валовой выпуск $i$-го продукта в основных ценах при росте конечного спроса на $j$-й продукт на 1 денежную единицу.

\section{Оценки мультипликативных әффектов в экономиках Китая и России}

Если обратиться к таблицам «Затраты Выпуск» по экономике Китайской народной республики за 2017 г., подготовленным Ази- 
атским банком развития ${ }^{12}$, можно увидеть, что исходные данные предоставлены в текущих ценах производителя (основных ценах) в миллионах долларов США, торговые и транспортные наценки не выделены, а, по всей видимости, содержатся в значениях выпуска по торговым и транспортным отраслям, отсутствуют чистые налоги на продукты, валовая добавленная стоимость по отрасли дается одним числом без разбиения на заработную плату, прибыль, амортизацию основного капитала и налоги, а также раздельно приводятся значения по отечественным и импортным товаром и услугам. Все перечисленное делает возможным применять предложенную в данной статье методику - формулы (4)-(16) - без дополнительной трансформации исходных данных.

${ }_{12}$ URL: https://data.adb.org/dataset/peoples-republicchina-input-output-economic-indicators
На рис. 1 представлены значения мультипликаторов валовой добавленной стоимости по каждой из отраслей $\left(M_{V A}\right)$, а также значения суммы по столбцам матричного мультипликатора валового выпуска $\left(M_{X}\right)$.

Анализируя рис. 2 , можно отметить, что значения отраслевых мультипликаторов лежат в диапазоне 4,8-6,1, что в общем и целом соответствует оценкам агрегированного мультипликатора Харрода, приведенным в табл. $1(5,26)$.

Если посмотреть на среднее арифметическое значение мультипликатора по товарам, то оно составляет 5,35, а по услугам5,70. Тут следует обратить внимание на то, что мультипликатор оценивает лишь потенциальное влияние экспорта, но не учитывает тот факт, есть ли и в каком объеме экспорт на самом деле. Так, максимальное значение мультипликатора у «Операций с недвижи-

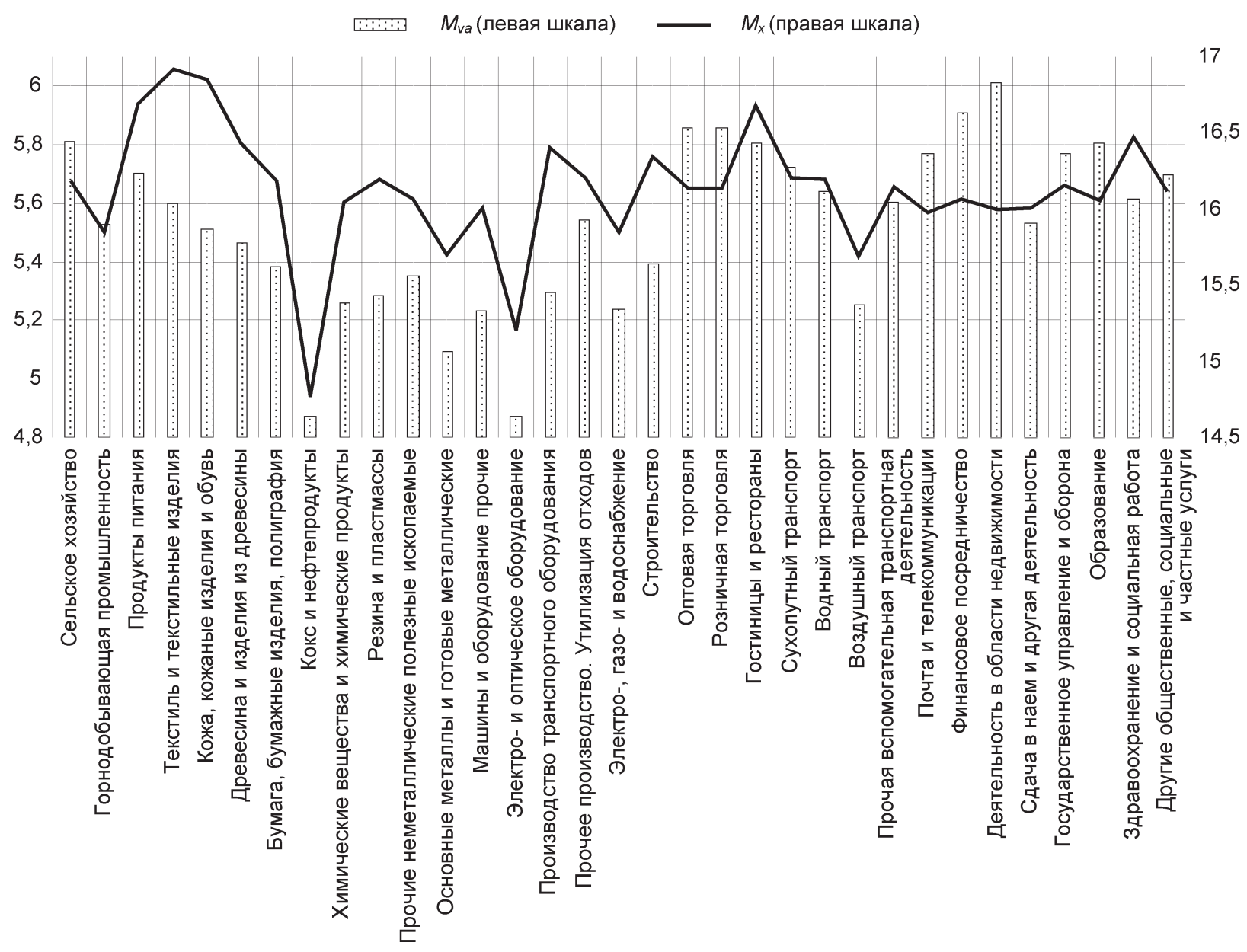

Рис. 2. Значения мультипликаторов валовой добавленной стоимости $\left(M_{V A}\right)$ и суммы по столбцам матричного мультипликатора валового выпуска $\left(M_{X}\right)$ для китайской экономики

Источник. Расчеты по таблицам «Затраты - Выпуск» Азиатского банка развития за 2017 г. 
мым имуществом» - 6,01, однако данный вид услуг не является объектом экспорта в принципе. С другой стороны, у ведущей экспортной отрасли «Электрическое и оптическое оборудование» (32,1\% в общем объеме экспорта в ценах производителя) мультипликатор равен всего лишь 4,87.

Довольно высокие значения мультипликатора в оптовой и розничной торговле $(5,86)$, а также у транспортных услуг $(5,25-5,72)$, однако следует понимать, что здесь речь, по всей видимости, идет в первую очередь о торговых и транспортных наценках, которые в реальной действительности «сидят» в ценах других товаров и услуг и напрямую зависят от объема продаж последних.

Обращение к таблицам «Затраты Выпуск» за 2016 г. по Российской Федерации показывает, что симметричная таблица отечественной продукции так же, как и в случае с данными по Китаю, может быть использована без дополнительной трансформации. Однако есть одно существенное отличие - сумма валовой добавленной стоимости дана с разбивкой на доходы домашних хозяйств (оплата труда), государства (налоги) и коммерческих организаций (амортизация и чистая прибыль), что позволяет определить элементы матриц валовой добавленной стоимости (доходов) $V$ и конечного потребления $C$ более детальным образом и тем самым оценить более тонкие эффекты мультипликативности.

В частности, в расчетах для российской экономики использовалась следующая спецификация матрицы $V$, имеющей в данном случае размерность $3 \times 98$, - элемент $j$ в каждой из трех строк матрицы определялся соответственно по одной из формул ниже (с учетом действующих в РФ ставок единого социального налога и налога на доходы физических лиц):

$$
\begin{aligned}
& v_{1 j}=\frac{w_{j}}{1,26 \cdot 1,13 \cdot x_{j}}, \\
& v_{2 j}=\frac{t_{j}^{A}+t_{j}^{B}+w_{j} \cdot\left(\frac{0,26}{1,26}+\frac{0,13}{1,26 \cdot 1,13}\right)}{x_{j}}, \\
& v_{3 j}=\frac{p r_{j}+a m_{j}}{x_{j}},
\end{aligned}
$$


Расчетные значения мультипликатора валовой добавленной стоимости (доходов) по отраслям с наибольшим вкладом в экспорт Российской Федерации по данным таблиц «Затраты - Выпуск» за 2016 г.

\begin{tabular}{|c|c|c|c|c|c|c|}
\hline \multirow[b]{3}{*}{ Отрасль } & \multirow{3}{*}{$\begin{array}{c}\text { Доля } \\
\text { в экспорте } \\
\text { (основные } \\
\text { цены), \% }\end{array}$} & \multicolumn{5}{|c|}{ Значение мультипликатора ВДС/доходов } \\
\hline & & \multirow{2}{*}{$\begin{array}{c}\text { без учета } \\
\text { дифферен- } \\
\text { циации } \\
\text { доходов }\end{array}$} & \multicolumn{4}{|c|}{ с учетом дифференциации доходов } \\
\hline & & & $\begin{array}{c}\text { Общее } \\
\text { значение }\end{array}$ & $\begin{array}{l}\text { Доходы } \\
\text { домохо- } \\
\text { зяйств }\end{array}$ & $\begin{array}{l}\text { Доходы } \\
\text { госу- } \\
\text { дарства }\end{array}$ & $\begin{array}{l}\text { Доходы } \\
\text { бизнеса }\end{array}$ \\
\hline $\begin{array}{l}\text { Нефть, включая нефть, получаемую } \\
\text { из битуминозных минералов; сланць } \\
\text { горючие (битуминозные) } \\
\text { и песчаники битуминозные }\end{array}$ & 18,5 & 4,33 & 3,90 & 0,96 & 0,50 & 2,44 \\
\hline Нефтепродукты & 12,9 & 4,22 & 3,86 & 0,99 & 0,52 & 2,35 \\
\hline $\begin{array}{l}\text { Услуги по оптовой торговле, } \\
\text { включая торговлю через агентов, } \\
\text { кроме услуг по торговле } \\
\text { автотранспортными средствами } \\
\text { и мотоциклами }\end{array}$ & 8,6 & 4,27 & 3,93 & 1,08 & 0,55 & 2,30 \\
\hline $\begin{array}{l}\text { Суда, летательные и космические } \\
\text { аппараты, прочие транспортные } \\
\text { средства и оборудование }\end{array}$ & 6,3 & 3,67 & 3,49 & 1,10 & 0,55 & 1,84 \\
\hline $\begin{array}{l}\text { Металлы основные драгоценные } \\
\text { и цветные прочие }\end{array}$ & 4,8 & 3,96 & 3,64 & 0,97 & 0,50 & 2,17 \\
\hline Вещества химические основные & 4,7 & 3,96 & 3,68 & 1,01 & 0,53 & 2,15 \\
\hline $\begin{array}{l}\text { Железо, чугун, сталь и ферросплавы, } \\
\text { трубы и элементы трубопроводные } \\
\text { соединительные, продукция } \\
\text { первичной обработки черных } \\
\text { металлов прочая }\end{array}$ & 4,6 & 3,89 & 3,62 & 1,03 & 0,53 & 2,06 \\
\hline $\begin{array}{l}\text { Прочие услуги, связанные } \\
\text { с предпринимательской } \\
\text { деятельностью }\end{array}$ & 3,1 & 4,15 & 3,89 & 1,17 & 0,59 & 2,14 \\
\hline $\begin{array}{l}\text { Газ природный в газообразном или } \\
\text { сжиженном состоянии, включая } \\
\text { услуги по сжижению } \\
\text { и регазификации природного газа } \\
\text { для транспортирования }\end{array}$ & 2,9 & 4,33 & 3,92 & 0,98 & 0,51 & 2,42 \\
\hline $\begin{array}{l}\text { Услуги воздушного и космического } \\
\text { транспорта }\end{array}$ & 2,5 & 3,36 & 3,16 & 0,91 & 0,47 & 1,78 \\
\hline
\end{tabular}

Источник. Авторская разработка.

чения отраслевых мультипликаторов практически в большинстве случаев несколько меньше (их значения лежат в диапазоне 2,37-4,53), но это вряд ли можно считать существенным различием (коэффициент корреляции между двумя наборами мультипликаторов - 0,96).

Если сравнивать указанные значения со значением агрегированного мультипликатора Харрода из табл. 1, то последний $(4,98)$ очевидно больше, но незначительно. Возможно, подобная разница возникает изза использования в первом случае национального дохода, а во втором - валовой добавленной стоимости с учетом чистых налогов на промежуточные продукты.
Отраслевые различия мультипликатора Харрода, по всей видимости, в первую очередь стоит объяснить тем, что импортоемкость отраслевого выпуска - главный фактор, который определяет значение мультипликатора. В тех отраслях, где ниже локализация отраслевого производства (и выше доля импортных комплектующих), мультипликатор меньше.

\section{Особенности учета косвенных налогов на продукты, транспортных и торговых наченок}

Если данные в таблицах «Затраты Выпуск» представлены таким образом, что выделены торговые и транспортные нацен- 
ки, а использование выпуска (по строкам) соответствующих торговых и транспортных отраслей уменьшено на их величину, то исходные таблицы не являются сбалансированными и непригодны для непосредственных расчетов. Кроме того, в этом случае может ставиться цель нахождения мультипликаторов, которые учитывают не основные цены, а цены покупателей. Здесь нужен более сложный подход для применения предложенной выше методики.

Следует отметить, что торговля и транспорт в системе «затраты-выпуск» всегда требовали специфического подхода. В терминологии Пазинетти они - особенные «отрасли» (peculiar «industires»), так как в исходных фактических данных описывающие их столбцы и строки матрицы прямых затрат на самом деле не содержат полноценных технических коэффициентов, а изначально включают в том числе и все потоки продукции, проходящие через торговлю и подвергающиеся транспортировке, включая торговые и транспортные наценки, включаемые в цены этой продукции (Pasinetti, 1977. PР. 41-42). Статистические манипуляции с исходными данными призваны устранить этот недостаток, однако они приводят к искажению реально наблюдаемой картины и для многих случаев прикладных расчетов необходимо осуществлять еще одну корректировку.

Подобное наблюдается в таблицах «Затраты - Выпуск» для белорусской экономики. В одной из прошлых публикаций (Пархименко, Быков, 2020) автором предлагалась пошаговая методика трансформации исходных данных белорусской официальной статистики в таблицы, в которых торговые и транспортные наценки «возвращены» в выпуск соответствующих отраслей ${ }^{13}$. Применение данной методики позволяет сбалансировать каждую из таблиц «Затраты - Выпуск» и осуществлять с ней корректные математические операции. В настоящей статье с учетом опыта практической апробации этой методики предлагается все торговые отрасли (строки/столбцы 39-41) и все транспортные отрасли

13 Подобное предлагают делать (без учета специфики белорусских таблиц «Затраты - Выпуск»), например, G.R. West и J. Randall. URL: htps://researchrepository.wvu.edu/ rri_pubs/115 (строки/столбцы 42-45) представлять в виде всего лишь двух агрегированных отраслей «Торговля» и «Транспорт».

Однако остается еще одна проблема корректный учет транспортных и торговых наценок в итоговых отраслевых мультипликаторах. Дело в том, что, анализируя выше влияние экспорта в случае Китая и России, предполагалось, что полученный мультипликатор нужно для прикладных оценок умножать на величину экспорта в основных ценах, тем самым все торговые и транспортные наценки, а также чистые налоги на продукты в цене покупателя остаются неучтенными и итоговая оценка эффекта может оказаться заниженной.

Для того чтобы рассчитанные по формуле (15) мультипликаторы валовой добавленной стоимости учитывали торговые и транспортные наценки, предлагается скорректировать их на дополнительную величину по формуле ${ }^{14}$ :

$$
\begin{aligned}
m v a_{j}^{\prime} & =m v a_{j}+m v a_{\text {торг }} \cdot \frac{s_{j}^{\text {торг }}}{x_{j}}+ \\
& +m v a_{\text {трансп }} \cdot \frac{s_{j}^{\text {трансп }}}{x_{j}},
\end{aligned}
$$

где $m v a_{j}, m v a_{j}^{\prime}-$ расчетный и скорректированный мультипликатор валовой добавленной стоимости по $j$-й отрасли;

$m v a_{\text {торг }}, m v a_{\text {трансп }}-$ расчетные мультипликаторы валовой добавленной стоимости по отраслям «Торговля» и «Транспорт»;

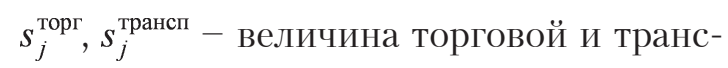
портной наценки на конечные товары и услуги по $j$-й отрасли;

$x_{j}-$ выпуск в основных ценах по $j$-й отрасли.

При использовании упрощенного подхода по валовой добавленной стоимости (см. пример расчетов по Китаю) полностью не учтенным остается такой элемент ВВП (при расчете производственным способом), как чистые налоги на товары и услуги. В расчетах по России - см. формулу (17) - часть этих налогов, учитываемых в промежуточном потреблении, принималась во внимание,

14 Данная формула завышает конечную оценку, так как относит всю совокупность торговых и транспортных наценок целиком на отечественные товары и услуги. 
однако налоги на конечную продукцию остались вне поля внимания.

Между тем в 2019 г. сумма таких налогов по отношению к сумме валовой добавленной стоимости по всем отраслям белорусской экономики составляла отнюдь не маленькую величину - 15\%. Если в расчетах мультипликативных эффектов это не учитывать как доходы государства, в дальнейшем идущие на государственные расходы, получаемые оценки будут довольно серьезно занижены.

Предлагается следующая методика оценки $j$-го элемента вектора-строки $V$ :

$$
\frac{g v a_{j}+t_{j}^{A}+t_{j}^{C} \cdot \frac{f_{j}^{\text {отеч }}}{f_{j}^{\text {отеч }}+f_{j}^{\text {имп }}}}{x_{j}},
$$

где - валовая добавленная стоимость по $j$-й отрасли;

$t_{j}^{C}-$ чистые налоги на конечные продукты по $j$-й отрасли;

$$
f_{j}^{\text {огеч }}, f_{j}^{\text {имп }}-\text { конечный спрос на отече- }
$$

ственные и импортные товары и услуги по $j$-й отрасли.

В табл. 3 представлены результаты расчетов по формулам (4)-(16) и (19), (20) для

Расчетные значения отраслевых мультипликаторов ВДС/ВВП по 20 отраслям

Таблица 3

\begin{tabular}{|c|c|c|c|c|}
\hline \multirow[b]{2}{*}{ Отрасль } & \multirow[b]{2}{*}{$\begin{array}{c}\text { Доля } \\
\text { в экспорте, } \\
\%\end{array}$} & \multicolumn{3}{|c|}{ Мультипликаторы } \\
\hline & & $\begin{array}{l}\text { только } \\
\text { по ВДС }\end{array}$ & $\begin{array}{c}\text { с учетом } \\
\text { чистых } \\
\text { налогов } \\
\text { на продукты }\end{array}$ & $\begin{array}{c}\text { с учетом } \\
\text { налогов, торговых } \\
\text { и транспортных } \\
\text { наценок }\end{array}$ \\
\hline Вещества химические и продукция химическая & 14,1 & 1,33 & 1,71 & 2,03 \\
\hline Кокс и нефтепродукты & 13,5 & 0,32 & 0,61 & 0,75 \\
\hline $\begin{array}{l}\text { Продукты пищевые, включая напитки, } \\
\text { и табачные изделия }\end{array}$ & $g l_{2}^{2} 2$ & 1,52 & 1,94 & 2,36 \\
\hline Транспорт & 8,9 & 1,66 & 1,91 & 1,91 \\
\hline $\begin{array}{l}\text { Услуги в области компьютерного } \\
\text { программирования, консультационные } \\
\text { и аналогичные услуги }\end{array}$ & 5,2 & 2,19 & 2,45 & 2,45 \\
\hline $\begin{array}{l}\text { Машины и оборудование, не включенные } \\
\text { в другие группировки, за исключением машин } \\
\text { для сельского и лесного хозяйства }\end{array}$ & 4,1 & 1,37 & 1,58 & 1,82 \\
\hline Металлы основные & 3,6 & 0,76 & 0,92 & 1,15 \\
\hline $\begin{array}{l}\text { Древесина и изделия из древесины и пробки, } \\
\text { кроме мебели; изделия из соломки } \\
\text { и материалов для плетения }\end{array}$ & 3,6 & 1,46 & 1,69 & 1,84 \\
\hline Автомобили, прицепы и полуприцепы & 3,4 & 1,00 & 1,26 & 1,64 \\
\hline Изделия резиновые и пластмассовые & 2,6 & 1,00 & 1,16 & 1,20 \\
\hline Оборудование электрическое & 2,6 & 1,00 & 1,20 & 1,48 \\
\hline $\begin{array}{l}\text { Текстиль и текстильные изделия, одежда, } \\
\text { меховые изделия }\end{array}$ & 2,4 & 1,41 & 1,72 & 2,16 \\
\hline $\begin{array}{l}\text { Изделия металлические готовые, кроме машин } \\
\text { и оборудования }\end{array}$ & 2,0 & 1,24 & 1,48 & 1,60 \\
\hline Мебель и прочая продукция & 2,0 & 1,41 & 1,76 & 2,39 \\
\hline Машины для сельского и лесного хозяйства & 1,8 & 1,40 & 1,60 & 1,72 \\
\hline $\begin{array}{l}\text { Здания и сооружения; работы } \\
\text { общестроительные по возведению зданий } \\
\text { и сооружений }\end{array}$ & 1,8 & 1,45 & 1,74 & 1,74 \\
\hline Сырая нефть и природный газ & 1,7 & 1,90 & 2,63 & 3,39 \\
\hline Изделия минеральные неметаллические прочие & 1,6 & 1,39 & 1,61 & 1,82 \\
\hline $\begin{array}{l}\text { Компьютеры, оборудование электронное } \\
\text { и оптическое }\end{array}$ & 1,6 & 1,18 & 1,40 & 2,08 \\
\hline Оборудование транспортное прочее & 1,3 & 1,07 & 1,31 & 2,08 \\
\hline
\end{tabular}
с наибольшим вкладом в экспорт Республики Беларусь, 2019 г.

Источник. Авторская разработка. 
белорусской экономики по данным таблиц «Затраты - Выпуск» за 2019 г. Следует отметить, что по отдельным отраслям налоги и наценки вносят существенный вклад: например, по коксу и нефтепродуктам мультипликатор ВВП составляет 233,3\% от мультипликатора ВДС, по прочему транспортному оборудованию - 194,6\%, по сырой нефти и природному газу - 178,3\%, по отрасли «Продукты пищевые, включая напитки, и табачные изделия» - 155,2\%, по отрасли «Вещества химические и продукция химическая» $-152,9 \%$.

Говоря про анализ в ценах покупателей, а не производителей, следует отметить, что торговые и транспортные наценки, а также чистые налоги на конечные продукты играют большую роль в динамике мультипликативных эффектов не только в случае отечественных товаров и услуг, но даже в случае конечного импорта. Априори предполагаемая в предлагаемой выше методике «утечка» средств на покупку конечного импорта на самом деле не является 100\%-й, так как конечные импортные товары и услуги облагаются налогами (чистые налоги на продукты), а также в их цену продажи входят торговые и транспортные наценки. Следовательно, даже при продаже конечных импортных товаров на внутреннем рынке государство и посреднические компании формируют свои доходы и не все деньги «выпадают» из оборота внутри экономики. Этот аспект, однако, в данной статьей остается вне рассмотрения и учета в методике расчетов.

В заключение отметим, что для прикладных макроэкономических расчетов ни мультипликатор Харрода в его статической версии, ни предложенные в статье матричные мультипликаторы в отраслевом разрезе не могут использоваться как справочные значения без должной осторожности. Это лишь ориентиры, показывающие потенциально возможный эффект тех или иных экспортных шоков при заданных допущениях.

Упоминаемое выше различие между мультипликаторами Туре I (например, полные затраты на единицу конечного спроса) и Tуре II (в отличие от первых учитывают вторичные эффекты, вызванные приростом доходов), как правило, считается нижней и верхней границами оценки фактического эффекта изменения конечного спроса (Oosterhaven, Piek, Stelder, 1986). При этом следует помнить, что мультипликаторы второго типа обычно учитывают лишь прирост доходов домохозяйств, что меньше прироста совокупного дохода, используемого в настоящей статье, и поэтому предлагаемые здесь оценки, вероятно, должны рассматриваться даже не как верхняя, а скорее - как максимально возможная граница.

Изменение цен, валютного курса и процентных ставок, противодействующие меры со стороны государства (например, стимулирование внутреннего спроса вместо «выпадающего» экспорта), поведение домохозяйств и инвесторов и другие упоминаемые ранее факторы - все это в реальной действительности будет приводить к значительно меньшим величинам реального эффекта экспортных шоков.

В любом случае предложенные мультипликаторы показывают «мощность» воздействия экспорта на экономику - будет ли это воздействие проявляться в количественной (объемы выпуска, добавленной стоимости, ВВП) или ценовой плоскости. Таким образом, для лиц, принимающих макроэкономические решения, становится возможным осуществлять ранжирование отраслей, на которые следует обращать первостепенное внимание в контексте экспортных шоков.

Для дальнейших исследований в области мультипликативных эффектов остается еще много открытых вопросов. Один из них - эконометрическая проверка наличия межотраслевых мультипликативных эффектов и соответствие их величины прогнозным оценкам. Еще один вопрос связан с тем, что, согласно подходу Харрода и предложенной в статье методике, потенциальные мультипликативные эффекты тем выше, чем меньше отношение импорта (а следовательно, и экспорта) к ВВП. Таким образом, более закрытые экономики потенциально должны сильнее ощущать последствия экспортных шоков. Кроме того, получается, что размер экономики никак не влияет на восприимчивость к колебаниям экспорта. Это противоречит сложившемуся пониманию процессов в мировой тор- 
говле и требует разъяснения или корректировки. Наконец, есть совокупность методических вопросов, например, насколько устойчивы во времени функции потребления для разных субъектов экономики и каким способом стоит оценивать коэффициенты потребления для минимизации ошибки прогноза или как учесть доходы от налогов и наценок, получаемые при продаже на внутреннем рынке импортных конечных продуктов.

\section{СПИСОК ЛИТЕРАТУРЫ (REFERENCES)}

Быков А.А., Пархименко В.А., Толкачев С.А. 2020. Влияние COVID-19 на российскую экономику: методологические подходы к оценке на основе межотраслевого баланса. Белорусский экономический журнал. № 2. С. 25-37. [Bykau A., Parkhimenka U., Tolkachev S. Influence of COVID19 on the Russian economy: Methodological approaches to the assessment based on the input-output tables. Belorusskiy ekonomicheskiy zhurnal. No 2. PP. 25-37. (In Russ.)]

Дондоков 3.Б.-Д., Булдаев А.С. 2013. Об одной модификации модели межотраслевого баланса. Вестник Бурятского государственного университета. № 9. C. 167-173. [Dondokov Z.B.-D., Buldaev A.S. 2013. On one modification of interbranch balance. Vestnik Buryatskogo gosudarstvennogo universiteta. No 9. PP. 167-173. (In Russ.)]

Ксенофонтов М.Ю., Широв А.А., Ползиков Д.А., Янтовский А.А. 2018. Оценка мультипликативных эффектов в российской экономике на основе таблиц «затраты-выпуск». Проблемы прогнозирования. № 2. C. 3-13. [Ksenofontov M.Yu., Shirov A.A., Polzikov D.A., Yantovsky A.A. 2018. Estimation of multiplier effects in the Russian economy based on input-output tables. Problemy prognozirovaniya. No 2. PP. 3-13. (In Russ.)]

Пархименко В.А., Быков А.А. 2020. Влияние изменения курса национальной валюты на макроэкономические показатели: оценка на основе методологии «Затраты - Выпуск». Белорусский экономический журнал. № 3. С. 58-73. [Parkhimenka U., Bykau A. 2020. Impact of changes in the national currency exchange rate on macroeconomic indicators: assessment based on the «input - output» methodology. Belorusskiy ekonomicheskiy zhurnal. No 3. PP. 58-73. (In Russ.)]

Ahiakpor J.C.W. 2001. On the Mythology of the Keynesian Multiplier: Unmasking the Myth and the Inadequacies of Some Earlier Criticisms. The American Journal of Economics and Sociology. Vol. 60. Iss. 4. PP. 745-773.

Araujo R.A., Lima G.T. 2007. A structural economic dynamics approach to balance-of-payments- constrained growth. Cambridge Journal of Economics. Vol. 31. Iss. 5. PP. 755-774.

Bairam E.I., Dempster G.J. 1991. The Harrod foreign trade multiplier and economic growth in Asian countries. Applied Economics. Vol. 23. PP. 1719-1724.

Gouvea R., Lima G. 2013. Balance-of-paymentsconstrained growth in a multisectoral framework: A panel data investigation. Journal of Economic Studies. Vol. 40. Iss. 2. PP. 240-254.

Gouvea R.R., Lima G.T. 2010. Structural Change, Balance-of-Payments Constraint and Economic Growth: Evidence from the Multi-Sectoral Thirlwall's Law. Journal of Post Keynesian Economics. Vol. 33. Iss. 1. PP. 169-204.

Gravino D. 2012. Economic and policy implications of industry interdependence: An Inputoutput approach. International Journal of Economics and Finance. Vol. 4. No 6. PP. 22-31.

Mankiw N.G. 2019. Macroeconomics. New York: Worth Publishers.

McCombie J.S.L. 1985. Economic growth, the Harrod foreign trade multipler and the Hicks' SuperMultiplier. Applied Economics. No 17. PP. 55-72.

Miller R.E., Blair P.D. 2009. Input-Output Analysis: Foundations and extensions. Cambridge: Cambridge University Press. 67 p.

Mitchell W., Wray L.R., Watts M. 2019. Macroeconomics. London: Red Globe Press.

Miyazawa K. 1960. Foreign trade multiplier, input-output analysis and the consumption function. Quarterly Journal of Economics. No 74. PP. 53-64.

Miyazawa K. 1968. Input-output analysis and interrelational multiplier as a matrix. Hitotsubashi Journal of Economics. No 7. PP. 39-58.

Miyazawa K. 1976. Input-Output Analysis and the Structure of Income Distribution. New York, Springer-Verlag.

Oosterhaven J., Piek G., Stelder D. 1986. Theory and practice of updating regional versus interregional interindustry tables. Papers in Regional Science. Vol. 59. Iss. 1. PP. 57-72.

Pasinetti L.L. 1977. Lectures on the Theory of Production. Columbia University Press.

Romero J., McCombie J.S.L. 2016. The multisectoral Thirlwall's law: evidence from 14 developed European countries using product level data. International Review of Applied Economics. Vol. 30. No 3. PP. 301-325.

Thirlwall A.P. 1979. The Balance of Payments Constraint as an Explanation of International Growth Rate Differences. Banca Nazionale del Lavoro Quarterly Review. Vol. 32. No 128. PP. 45-53.

Thirlwall A.P. 2012. Balance of Payments Constrained Growth Models: History and Overview. In: Soukiazis E., Cerqueira P.A. (Eds.) Models of Balance of Payments Constrained Growth. Palgrave Macmillan, London.

Trigg A., Ricardo A. 2014. A Multi-sectorial Assessment of the Static Harrod Foreign Trade Multiplier. MPRA Paper. URL: https://mpra.ub.unimuenchen.de/53242/ 
In citation: Belorusskiy Ekonomicheskiy zhurnal. 2021. No 3. PP. 40-57.

Belarusian Economic Journal. 2021. No 3. PP. 40-57.

\section{ASSESSMENT OF INPUT-OUTPUT MULTIPLICATIVE EFFECTS CAUSED BY EXPORT SHOCKS}

\section{Uladzimir Parkhimenka ${ }^{1}$}

Author affiliation: ${ }^{1}$ Belarusian State University of Informatics and Radioelectronics (Minsk, Belarus). Corresponding author: Uladzimir Parkhimenka (parkhimenko@bsuir.by).

ABSTRACT. The article suggests a methodology for assessing the multiplier effects caused by export shocks is proposed, based on Leontiev's input-output methodology and Harrod's concept of a foreign trade multiplier. The methodology allows taking into account the specifics of the expenses of households, the state and commercial organizations, as well as making estimates of both basic and purchasers' prices. Evaluation of multiplicative effects iteratively is allowed. The methodology was tested within the framework of assessing the sectoral multipliers of gross output, gross value added and GDP for Chinese, Russian and Belarusian economies.

KEYWORDS: input-output model, input-output methodology, Harrod's multiplier, multiplication effects in the economy, export shocks.

JEL-code: C50, C67, E12, E17.

DOI: $10.46782 / 1818-4510-2021-3-40-57$

Received 30.07.2021 